encountering a wide range of genetic conditions and issues. The tables are well laid out and helpful though obviously not fully comprehensive. Illustrations are sparse but relevant. Suggested reading at the end of each chapter is reasonable and up to date in 1993! This handbook can be usefully used to complement other genetic counselling texts. It is a book that I would recommend to any clinical library or department as a helpful introduction to the appreciation and understanding of the genetic contribution to clinical disease.

\section{S J DAVIES}

Guide to Techniques in Mouse Development. Methods in Enzymology Volume 225. Editors Paul $M$ Wassarman, Melvin L DePamphilis. (Pp 1021; \$59.95.) New York: Academic Press. 1993.

In the preface to this impressive book, the editors remark that its publication is timely in view of the enormous and rapidly growing interest in the mouse as an experimental organism. Perhaps the size of the book is also an indication of this explosion of knowledge.

When I first became interested in mouse embryology in the early 1970 s, the mammalian embryologists "bible" was Methods in experimental embryology of the mouse by Keen A Rafferty Jr. This excellent book consisted of two sections, an appendix, and 94 pages. As well as dealing with basic embryo culture and handling, there were instructions for modish 1970s techniques such as aggregation chimeras, in vitro fertilisation, and artificial insemination.

In the 1980 s, Rafferty was complemented and extended by the publication of $\mathrm{Ma}$ nipulating the mouse embryo by Brigid Hogan, Frank Costantini, and Elizabeth Lacy. This book moved things on by an order of magnitude, consisting of eight sections, three appendices, and 332 pages. We now had detailed information on the construction of transgenic animals and their analysis, also the production of embryonic stem (ES) cell lines, and the formation of chimeras by their injection into blastocysts.

We are now in the 1990s and looking at the latest brave attempt to encapsulate all that is known about experimental mouse embryology into one comprehensible volume. Guide to techniques in mouse development contains 12 sections, 56 chapters, and 1021 pages. There is an incredible amount of detailed information here and I feel it will be a daunting task for an editor in the next decade to supersede this volume.

Section I is titled "General methodology" and deals with mouse breeding records and anaesthesia. Section II is "Germ cells and embryos" and begins with chapters on the isolation, culture, and manipulation of primordial germ cells. We then progress to the isolation, handling, and culture of oocytes, sperm, pre- and postimplantation embryos.

Section III considers various aspects of fertilisation including micromanipulation of gametes and embryos, in vitro capacitation and in vitro fertilisation. The next four sections discuss gene expression from several different angles: messenger RNA, reporter genes, proteins, and methylation.

Section VIII is concerned with gene identification and has chapters dealing with the construction of cDNA libraries and yeast artificial chromosomes (YACS). There are also chapters on genome mapping, cloning of mutations, and insertional mutagenesis by retroviruses and promotor traps in ES cells.

The next three sections deal with nuclear transplantation, production of transgenic animals by pronuclear injection, embryonic stem cells, and gene targeting. In short, there is something for everybody in this book, but I must say that the great swathes of in formation are not presented with the exceptional clarity contained in Manipulating the mouse embryo by Brigid Hogan et al. Indeed, some of the chapters resemble research publications rather than a laboratory manual. This is a small criticism, however, and I think merely reflects how large a subject the study of mouse development has become. The range of topics covered and the depth in which most have been treated is very impressive and the editors deserve to be congratulated on the completion of what must have been a mammoth task. This book deserves to be in the laboratory of every developmental biologist.

\section{PETER H GLENISTER}

The Phenotypic Mapping of Down Syndrome and Other Aneuploid Conditions. Editor Charles J Epstein. Proceedings of a National Society Conference held in New York, January 1993. (Pp 238; \$96.00.) New York: Wiley-Liss. 1993.

This volume contains papers presented at a conference held early in 1993; the theme of the conference was to correlate the phenotype of conditions with human aneuploidy with the trisomic or monosomic chromosomal material.

The editor, Dr Charles J Epstein, collected and edited a number of excellent review papers that are of interest to clinical and molecular geneticists. The chapter on the conceptual bases for the phenotype mapping of conditions resulting from aneuploidy by Epstein should be read and appropriately filed by all those who create phenotypic/genotypic maps of aneuploidies. It reflects the experience and long term thinking of the author of how to correlate a particular chromosomal region with a particular phenotype of aneuploidy. The second chapter by Schinzel gives an extensive and very useful catalogue of karyotype-phenotype correlations in autosomal chromosomal aberrations. The chapter on physical mapping of chromosome 21 , from the laboratory of Patterson, discusses some aspects of the physical mapping of this chromosome and in particular the use of somatic cell hybrids and the joint YAC screening for the completion of the physical mapping. The numerous contributions of the Patterson laboratory to chromosome 21 mapping are included in this chapter. The chapter by Haines on the genetic linkage map of chromosome 21 provides data obtained from the Venezuelan families. In addition, it gives a biological flavour to the linkage map. The two chapters from the Korenberg and the Sinet and Delabar laboratories give a detailed account of the molecular mapping of several phenotypes of Down's syndrome on chromosome 21. The two chapters are written in different style and their conclusions are slightly divergent. The Korenberg emphasis is against the definition of a single "Down syndrome minimal region", while the SinetDelabar paper puts more emphasis on the importance of the D21S55 region as being central for many phenotypes of the syndrome. It is my impression that both studies concluded that different parts of the chromosome are associated with different elements of the Down's syndrome phenotype. The chapter by Davisson and collaborators on the production of a segmental trisomy 16 mouse as a model for Down's syndrome is, I think, the one paper to xerox and keep from the book, since it contains important information not published elsewhere. The chapter is written with such attention to detail that it looks like an important original paper in an outstanding journal. It describes years of hard work and the first systematic analysis of this extremely valuable mouse model. The contribution from Groner's laboratory on gene dosage effects of PFKL on transfected PC12 cells emphasises in a detailed, systematic, and convincing way the importance of studying the gene dosage in cultured cells.

The remaining five chapters deal with problems of aneuploidy other than trisomy 21 . Wilson discussed the karyotype/phenotype correlation of trisomy 18 and Overhauser gave a molecular analysis of $18 \mathrm{q}$ deletions which provide an outstanding tool in further mapping studies on this chromosome. Emanuel and collaborators contributed a nice chapter on the recent and exciting developments of chromosome 22 microdeletion syndromes including DiGeorge and VCF syndromes. Lalande and collaborators enriched the book with a short chapter on the mapping of the regions involved in the Angelman and PraderWilli syndromes. Finally, the paper by Patel, Lupski, and collaborators gave a thorough discussion of the exciting findings of the duplication of chromosome $17 p$ that includes the PMP22 gene, which is responsible for Charcot-Marie-Tooth disease type $1 \mathrm{~A}$ and probably represents the best example to study the effects of a short segment chromosomal imbalance. Their chapter provides a number of important conclusions to investigators who are confronted with the question of gene dosage and its relation to disease.

In summary, this volume contains an excellent collection of papers on phenotype/ genotype correlation of aneuploidies. Of course, it suffers from all the symptoms of conference proceedings; however, some of the chapters are original, not published elsewhere, and useful to the investigator of human aneuploidies.

S ANTONARAKIS 